\title{
Pseudo-holomorphic curves: A very quick overview
}

https://doi.org/10.1515/coma-2020-0105

Received June 25, 2020; accepted October 11, 2020

Abstract: This is a review article on pseudo-holomorphic curves which attempts at touching all the main analytical results. The goal is to make a user friendly introduction which is accessible to those without an analytical background. Indeed, the major accomplishment of this review is probably its short length.

Nothing in here is original and can be found in more detailed accounts such as [6] and [8]. The exposition of the compactness theorem is somewhat different from that in the standard references and parts of it are imported from harmonic map theory [7], [5]. The references used are listed, but of course any mistake is my own fault.

Keywords: Pseudo-holomorphic curves

MSC: Primary: 32Q65, Secondary: 53B35, 53C15, 53C38, 53D05, 58J60

\section{Basic definitions and geometric properties}

\subsection{Definitions}

Let $\left(M^{2 n}, \omega\right)$ be a compact symplectic manifold with a compatible almost complex structure $J$ and associated metric $g$. This means that $g(\cdot, \cdot)=\omega(\cdot, J \cdot)$ and $J^{\star} \omega=\omega$, where $J$ acts on $\omega$ by pullback.

Given a Riemann surface $(\Sigma, j)$, a map $u:(\Sigma, j) \rightarrow(M, J)$ is said to be pseudo-holomorphic if it satisfies

$$
\bar{\partial}_{J} u:=\frac{1}{2}\left(d u+J_{u} \circ d u \circ j\right)=0,
$$

or in other words $J_{u} \circ d u=d u \circ j$, i.e. $d u$ is a complex linear map. We shall refer to this as the Cauchy-Riemman equation and regard the Cauchy-Riemann operator

$$
\bar{\partial}_{J}: C^{\infty}(\Sigma, M) \rightarrow \mathcal{T},
$$

as a section of the bundle $\mathcal{T} \rightarrow C^{\infty}(\Sigma, M)$ whose fiber at $u \in C^{\infty}(\Sigma, M)$ is $\mathcal{T}_{u}=C^{\infty}\left(\Sigma, \Lambda^{0,1} \Sigma \otimes u^{\star} T M\right)$.

Remark 1 (Cauchy-Riemman equation locally). Using a local holomorphic coordinate $z=x+i y$ on $\Sigma$ we have $j\left(\partial_{x}\right)=\partial_{y}$ and so the Cauchy-Riemann equation turns into

$$
\frac{\partial u}{\partial x}=-J \circ \frac{\partial u}{\partial y}
$$

where $\frac{\partial u}{\partial x}=d u\left(\partial_{x}\right)$.

Notation 1. All Riemann surfaces denoted by $(\Sigma, j)$, or simply $\Sigma$, will be assumed to be closed (compact without boundary) unless otherwise stated.

^Corresponding Author: Gonçalo Oliveira: Universidade Federal Fluminense IME-GMA, Niterói, Brazil, E-mail: galato97@gmail.com 


\subsection{Energy}

The harmonic map energy of a map $u:(\Sigma, j) \rightarrow(M, J)$ is given by

$$
\mathcal{E}(u):=\int_{\Sigma}|d u|^{2} \operatorname{vol}_{g_{\Sigma}},
$$

which in order to define the norm $|d u|$ uses both $g$ and a compatible metric $g_{\Sigma}$ on $\Sigma$. Here, by a metric $g_{\Sigma}$ on $\Sigma$ being compatible we mean to say it induces the same Riemann surface structure as $j$.

Remark 2 (The energy density does not depend on $g_{\Sigma}$ ). In a local holomorphic coordinate $z$ we have $g_{\Sigma}=$ $f(x, y)|d z|^{2}$ for some positive function $f$. From this one easily computes

$$
|d u|^{2} \operatorname{vol}_{g_{\Sigma}}=\left(\left|\partial_{x} u\right|_{g}^{2}+\left|\partial_{y} u\right|_{g}^{2}\right) d x \wedge d y,
$$

and notice that $\left|\partial_{x} u\right|_{g}$ and $\left|\partial_{x} u\right|_{g}^{2}$ only depend on the metric $g$ on M. From this formula it then follows that $\mathcal{E}(u)$ does not depend on the choice of $g_{\Sigma}$ but only on its conformal class, which is fixed by $j$.

The following result shows that pseudo-holomorphic maps minimize the harmonic map energy amongst maps with homologous image.

Proposition 1 (Energy identity and minimization). For any $u: \Sigma \rightarrow M$ we have

$$
\varepsilon(u)=\int_{\Sigma} 2\left|\bar{\partial}_{J} u\right|^{2} \operatorname{vol}_{g_{\Sigma}}+\int_{\Sigma} u^{*} \omega .
$$

Notice that the second term only depends in the homology class $[u(\Sigma)] \in H_{2}(M)$ while the first is nonnegative and vanishes if and only if $u$ is pseudo-holomorphic. Hence, any pseudo-holomorphic map minimizes $\mathcal{E}$ amongst maps $u$ with homologous image.

Proof. In a local holomorphic coordinate $z=x+i y$ in $\Sigma$ we have $j d x=-d y$ and $j d y=d x$. Thus, $2 \bar{\partial}_{J} u=$ $\left(\partial_{x} u+J_{u} \partial_{y} u\right) d x+\left(\partial_{y} u-J_{u} \partial_{x} u\right) d y$ from which we compute

$$
\begin{aligned}
\mathrm{u}_{\left|\bar{\partial}_{J} u\right|^{2} \mathrm{vol}_{\Sigma}} & =\left(\left|\partial_{x} u+J_{u} \partial_{y} u\right|_{g}^{2}+\left|\partial_{y} u-J_{u} \partial_{x} u\right|_{g}^{2}\right) d x \wedge d y \\
& =2\left(\left|\partial_{x} u\right|_{g}^{2}+\left|\partial_{y} u\right|_{g}^{2}+\left\langle\partial_{x} u, J_{u} \partial_{y} u\right\rangle_{g}-\left\langle\partial_{y} u, J_{u} \partial_{x} u\right\rangle_{g}\right) d x \wedge d y \\
& =2\left(\left|\partial_{x} u\right|_{g}^{2}+\left|\partial_{y} u\right|_{g}^{2}-\omega\left(\partial_{x} u, \partial_{y} u\right)-\omega\left(\partial_{x} u, \partial_{y} u\right)\right) d x \wedge d y \\
& =2|d u|^{2} \operatorname{vol}_{\Sigma}-2 \omega(d u \wedge d u),
\end{aligned}
$$

where we have used remark 2 and the compatibility of $(\omega, J, g)$. The result follows immediately from passing term $\omega(d u \wedge d u)=u^{\star} \omega$ to the other side, diving by 4 , and integrating over $\Sigma$.

\subsection{Pseudo-holomorphic curves are calibrated}

A symplectic form $\omega$ has the property that for any compatible $J$ and 2-plane $V$ we have $\left.\omega\right|_{V} \leq \operatorname{vol}_{V}$, where $\operatorname{vol}_{V}$ is the volume form on $V$ associated with the compatible metric $g(\cdot, \cdot)=\omega(\cdot, J \cdot)$. Moreover, equality is achieved if and only if $V$ is complex. This property, known as Wirtinger's inequality, is enough to show that pseudoholomorphic curves minimize, not only $\varepsilon$, but also the area functional in their homology class. Indeed, let $u: \Sigma \rightarrow M$ be a pseudo-holomorphic curve and $\tilde{\Sigma} \subset M$ a curve homologous to $u(\Sigma)$. Then, there is a cobordism $N^{3} \subset M$ from $\tilde{\Sigma}$ to $u(\Sigma)$ and as $\omega$ is closed we find that $0=\int_{N} d \omega=\int_{\tilde{\Sigma}} \omega-\int_{u(\Sigma)} \omega$. Using this we compute

$$
\operatorname{Area}(\tilde{\Sigma})=\int_{\tilde{\Sigma}} \operatorname{vol}_{\tilde{\Sigma}} \geq \int_{\tilde{\Sigma}} \omega=\int_{u(\Sigma)} \omega=\int_{u(\Sigma)} \operatorname{vol}_{u(\Sigma)}=\operatorname{Area}(u(\Sigma)),
$$

which shows that $u(\Sigma)$ minimizes area in its homology class. We shall state this here for completeness. 
Proposition 2 (Area minimization property). Let $(M, \omega, J)$ be a symplectic manifold equipped with a compatible almost complex structure and $u: \Sigma \rightarrow M$ a pseudo-holomorphic curve ${ }^{1}$. Then, $u(\Sigma)$ minimizes area in its homology class.

As a closing remark, we simply summarize the conclusions we arrived at in two sentences. Pseudoholomorphic curves, are a very special kind of harmonic maps with their image being very special minimal surfaces. They minimize both functionals Area and $\mathcal{E}$, having in fact

$$
\operatorname{Area}(u(\Sigma))=\varepsilon(u)
$$

\section{Consequences of the minimal surface point of view}

\subsection{The monotonicity formula}

A key property of holomorphic curves which is a shadow of them being minimal surfaces is the monotonicity formula. Below we give a "calibrated" proof of this property which the author learned in a talk from Simon Donaldson.

Proposition 3 (Monotonicity). Let $(\Sigma, j)$ be a Riemann surface with a compatible metric $g_{\Sigma}$ and $(M, \omega, J) a$ symplectic manifold $u: \Sigma \rightarrow M$ equipped with an almost complex structure. Then, there is $R>0$ such that: for all $x \in u(\Sigma)$ and $p$-holomorphic map $u: \Sigma \rightarrow M$, the quantity

$$
\frac{1}{r^{2}} \int_{u^{-1}\left(B_{r}(x)\right)}|d u|^{2} \operatorname{vol}_{\Sigma}
$$

is a non-decreasing function of $r \in(0, R)$.

Proof for the flat metric on $M=\mathbb{C}^{n}$. As $u$ is a p-holomorphic map we may choose $g_{\Sigma}$ to be the induced metric $\left.g\right|_{\Sigma}$. Furthermore, we may as well suppose with no loss of generality that $u(x)=0$ in which case we shall write $B_{r}:=B_{r}(0)$. Then, it follows from the energy identity that $|d u|^{2} \operatorname{dvol}_{\Sigma}=u^{\star} \omega$ and

$$
f(r):=\frac{1}{r^{2}} \int_{u^{-1}\left(B_{r}\right)} u^{\star} \omega=\frac{1}{r^{2}} \int_{B_{r} \cap u(\Sigma)} \omega=\frac{1}{r^{2}} \operatorname{Area}\left(B_{r} \cap u(\Sigma)\right) .
$$

From this, and writing $\omega=d \theta$ with $\left|\theta_{p}\right| \leq|p|$ we find

$$
f^{\prime}(r)=-\frac{2}{r^{3}} \operatorname{Area}\left(B_{r} \cap u(\Sigma)\right)+\frac{1}{r^{2}} \operatorname{Length}\left(\partial B_{r} \cap u(\Sigma)\right),
$$

and so it will be enough to prove that

$$
r \text { Length }\left(\partial B_{r} \cap u(\Sigma)\right) \geq 2 \text { Area }\left(B_{r} \cap u(\Sigma)\right) .
$$

To do this recall that for $\rho(\cdot)=|\cdot|$, we have $L_{\rho \partial_{\rho}} \omega=2 \omega$ and using $\left|\iota_{\partial \rho} \omega\right|=1$ we have

$$
\text { 2Area }\left(B_{r} \cap u(\Sigma)\right)=\int_{B_{r} \cap u(\Sigma)} d\left(\iota_{\rho} \partial_{\rho} \omega\right)=r \int_{\partial B_{r} \cap u(\Sigma)} \iota_{\partial_{\rho}} \omega \leq r \text { Length }\left(\partial B_{r} \cap u(\Sigma)\right),
$$

as we wanted to prove.

1 recall that we always assume $\Sigma$ is compact 
Remark 3 (Minimal surfaces point of view and non-squeezing). In the theory of minimal surfaces, the quantity 2.1 is more usually written as

$$
\frac{1}{r^{2}} \operatorname{Area}\left(u(\Sigma) \cap B_{r}(x)\right) .
$$

Then, the statement is that this quantity is a non-decreasing function of $r \in(0, R)$. In particular, the limit of this quantity as $r \rightarrow 0$ exists and letting $c$ be this limit we find that

$$
\operatorname{Area}\left(u(\Sigma) \cap B_{r}(x)\right) \geq c r^{2} .
$$

This property was one of the key ingredients used by Gromov in his proof of the nonsequeezing theorem which we shall state and prove in 7 . The main idea being that if a symplectic embedding $i: B_{R}^{2 n} \hookrightarrow C_{r}^{2 n}=D_{r}^{2} \times \mathbb{R}^{2 n-2}$ was to exist for $R>r$, then the radius $R$-ball would symplectically fit into $S_{r+\delta}^{2} \times \mathbb{T}^{2 n-2}$ where $r+\delta<R$. One then argues that it is possible to find a pseudo-holomorphic sphere $C$ passing through $i(0)$ with image in the class $\left[S_{r+\delta}^{2} \times\{0\}\right]$ which then has area (and energy) $\pi(r+\varepsilon)^{2}$. However, then $i^{-1}(C)$ is a minimal surface passing through the origin which implies that its area is at least that of the disk through the origin, i.e. $\pi R^{2}>\pi(r+\delta)^{2}$, leading to a contradiction. Of course, the details of the argument are more involved than in this short sketch. They key point missing resides in proving that such a pseudo-holomorphic curve does exist.

\subsection{Removable singularities}

The monotonicity property and the minimal surface viewpoint may also be used in proving a removable singularity theorem. Here we state the most general result which asserts that the extended map is smooth, but only prove continuity (the proof here follows that in [6]).

Proposition 4 (Removal of singularities). Let $(M, \omega, J)$ be a compact symplectic manifold equipped a compatible almost complex structure. Then, any finite energy pseudo-holomorphic curve $u: D^{2} \backslash\{0\} \rightarrow M$ smoothly extends over $\{0\}$.

Proof of continuity. Suppose not, i.e. that $u$ does not continuously extend over $\{0\}$, then it has different accumulation points as $z \rightarrow 0$, say $p_{1} \neq p_{2} \in M$. Let $\delta<\operatorname{dist}\left(p_{1}, p_{2}\right) / 3$, we shall first prove that both $u^{-1}\left(B_{\delta}\left(p_{1}\right)\right)$ and $u^{-1}\left(B_{\delta}\left(p_{2}\right)\right)$ have a finite number of connected components. Indeed, any such component $C$ of $u^{-1}\left(B_{\delta}\left(p_{1}\right)\right)$ is such that

$$
\left.u\right|_{C}: C \rightarrow M,
$$

is a minimal surface passing arbitrarily close to the point $p_{1} \in M$. Thus, by the monotonicity formula

$$
\operatorname{Area}\left(u(C) \cap B_{\delta}\left(p_{1}\right)\right) \geq c \delta^{2},
$$

for some constant $c$ only depending on the target geometry. In particular, as $C \subset \Sigma$ and $\mathcal{E}(u)=\operatorname{Area}(u(\Sigma))$ is finite by hypothesis, we find that there can be at most a finite number of such components. Thus, $u^{-1}\left(B_{\delta}\left(p_{1}\right)\right)$ and $u^{-1}\left(B_{\delta}\left(p_{2}\right)\right)$ have a finite number of components and so there is a connected component of each of these containing 0 as an accumulation point. From this we conclude that for sufficiently small $r>0$ the circles $\partial D_{r}$ intersect both these components and so $u\left(\partial D_{r}\right)$ meets both $B_{\delta}\left(p_{1}\right)$ and $B_{\delta}\left(p_{2}\right)$. As $\operatorname{dist}\left(p_{1}, p_{2}\right)>3 \delta$ we have Length $\left(u\left(\partial D_{r}\right)\right) \geq \delta$ for all small $r$. Furthermore, as $u$ is holomorphic

$$
\frac{\partial u}{\partial r}+\frac{1}{r} J \frac{\partial u}{\partial \theta}=0,
$$

and so $\left|\partial_{r} u\right|=r^{-1}\left|\partial_{\theta} u\right|$. Hence, the energy of $u$ is

$$
\mathcal{E}(u)=\int_{D} \frac{\left|\partial_{\theta} u\right|^{2}}{r^{2}} r d r \wedge d \theta \geq \int_{0}^{1}\left(\int_{0}^{2 \pi}\left|\partial_{\theta} u\right| d \theta\right)^{2} \frac{d r}{r} \geq \int_{0}^{1} \frac{\delta^{2}}{r} d r=\infty,
$$

contradicting the assumption that $u$ has finite energy. 
Remark 4. The idea of the previous proof can be basically summarized as follows. The fact that $u(D \backslash\{0\})$ is a minimal surface forces the circles $u\left(\partial D_{r}\right)$ to not get very far apart from each other. The, mechanism by which this happens can be understood from the strategy of the proof. If $u(z)$ has different limit points as $z \rightarrow 0$, the circles $u\left(\partial D_{r}\right)$ would be very long which would force $u$ to have infinite energy.

\section{Intuition for main compactness result}

This section explain the main intuition behind the failure of a family of pseudo-holomorphic curves to converge to another one in a nice manner. Our exposition is motivated by that in [3].

\subsection{Comparison with geodesics}

Geodesics in a Riemannian manifold $(M, g)$ locally minimize length and are critical points of the energy functional

$$
\varepsilon(\gamma)=\int_{a}^{b}|\dot{\gamma}(d t)|^{2} d t
$$

for $\gamma:[a, b] \rightarrow M .^{2}$ From the fundamental theorem of calculus and the Cauchy-Schwarz (or Hölder) inequality we find that for any $s_{1}, s_{2} \in[a, b]$

$$
\operatorname{dist}\left(\gamma\left(s_{2}\right), \gamma\left(s_{1}\right)\right) \leq \int_{s_{1}}^{s_{2}}|\dot{\gamma}(t)| d t \leq\left|\int_{s_{1}}^{s_{2}} d t\right|^{1 / 2}\left(\int_{s_{1}}^{s_{2}}|\dot{\gamma}(t)|^{2} d t\right)^{1 / 2},
$$

which in terms of the energy we can rewrite as

$$
\frac{\operatorname{dist}\left(\gamma\left(s_{2}\right), \gamma\left(s_{1}\right)\right)}{\left|s_{2}-s_{2}\right|^{1 / 2}} \leq \mathcal{E}(\gamma)^{1 / 2} .
$$

As this is valid for all $s_{2}, s_{1} \in[a, b]$ we conclude that a bound on the energy yields a bound on the $C^{0, \frac{1}{2}}$-norm of $\gamma:[a, b] \rightarrow M$. Now let $\left\{\gamma_{i}\right\}_{i \in \mathbb{N}}$ be a family of curves with uniformly bounded energy $\mathcal{E}\left(\gamma_{i}\right) \leq C$, then the $C^{0, \frac{1}{2}}$-bound obtained in each member of the family is uniform. This norm is sufficiently strong to guarantee the equicontinuity of the family $\left\{\gamma_{i}\right\}_{i \in \mathbb{N}}$ and so there is a subsequence which uniformly converges a curve $\gamma_{\infty}:[a, b] \rightarrow M$.

The same compactness phenomenon does not generalize to dimensions greater than one. Indeed we can suspect that to be the case already from the failure of the embedding $L_{1}^{2} \rightarrow C^{0}$ in dimension 2 as the next example shows.

Example 1 (Failure of borderline Sobolev embedding). For $r=\sqrt{x^{2}+y^{2}}$, the function $f(r)=\log \log r^{-1}$ in $\mathbb{D}_{\frac{1}{2}} \subset \mathbb{R}^{2}$ has square integrable derivative, i.e. energy or $L_{1}^{2}$-norm, but is unbounded and so not in $C^{0}$. Indeed, $f^{\prime}(r)=\frac{1}{r \log r}$ and

$$
\int_{\mathbb{D}_{1 / 2}}\left|f^{\prime}(r)\right|^{2} \operatorname{vol}_{\mathbb{R}^{2}}=2 \pi \int_{0}^{1 / 2} \frac{1}{r(\log r)^{2}} d r=-\left.\frac{2 \pi}{\log r}\right|_{r=0} ^{1 / 2}<+\infty .
$$

From the point of view of a family of pseudo-holomorphic maps $\left\{u_{k}\right\}_{k \in \mathbb{N}}$, with $u_{k}: \Sigma \rightarrow M$, the failure of this Sobolev embedding suggests that an energy bound may not be enough to guarantee the existence of a uniformly convergent subsequence. That is indeed the case as we shall see in the next example.

2 Notice that this is the exact analogue of the harmonic map energy we wrote down in 2-dimensions. The length, on the other hand, is given by Length $(\gamma)=\int_{a}^{b}|\dot{\gamma}(t)| d t$. 


\subsection{Bubbling example}

Let $\Sigma=\mathbb{C P}^{1}=M$ with the Fubini-Study structures which in homogeneous coordinates $[1: z] \in \Sigma$ and $[1: w] \in M$ we write as

$$
\omega_{\Sigma}=\frac{i}{2} \frac{d z \wedge \overline{d z}}{\left(1+|z|^{2}\right)^{2}}, \quad \omega_{M}=\frac{i}{2} \frac{d w \wedge \overline{d w}}{\left(1+|w|^{2}\right)^{2}} .
$$

Then, we let $\tilde{u}_{\infty}: \mathbb{C} \rightarrow \mathbb{C}$ be a rational map which when written as a quotient of two polynomials $\frac{p(z)}{q(z)}$ with no common factors with $q$ having degree $n$. We will further suppose that $u_{\infty}(\infty)=0$ (this amounts to assuming $\operatorname{deg}(q)>\operatorname{deg}(p))$ and that $q(0) \neq 0$. This extends to a rational map which we interpret as $u_{\infty}: \Sigma \rightarrow M$. Then, $\mathcal{E}\left(u_{\infty}\right)=\pi n$. Then, we consider the maps

$$
u_{k}([1: z])=\left[1: 1 / k z+\tilde{u}_{\infty}(z)\right]
$$

which also satisfy $u_{k}(\infty)=0$. Our assumptions on $p$ and $q$ imply that these maps $u_{k}$ have degree $n+1$, i.e. they have energy $\pi(n+1)$. It is easy to see that, away from the point $0=[1: 0]$, the maps $u_{k}$ uniformly converge with all derivatives to $u_{\infty}$. We see here the following teo phenomena:

(a) Convergence away from the blow up set $S=\{0\}$, meaning that $u_{k} \rightarrow u_{\infty}$ uniformly with all derivatives on compact subsets of $\Sigma \backslash S$, and

$$
\mathcal{E}\left(u_{\infty}\right) \leq \mathcal{E}\left(u_{k}\right)
$$

(b) Removal of singularities, meaning that the limiting map $u_{\infty}: \Sigma \backslash S \rightarrow M$ smoothly extends over the points of $S$.

However, notice that in the example above $u_{\infty}$ has degree $n$ and so energy

$$
\varepsilon\left(u_{\infty}\right)=\pi n<\mathcal{E}\left(u_{\infty}\right)=\pi(n+1) .
$$

This raises the question: where did the extra $\pi$ of energy go? The answer is roughly that as $k \rightarrow+\infty$ it got concentrated at 0 and escaped (or bubbled off) through there. To understand this we compute the energy density at $z=0$. Indeed, using the auxiliar map $v_{k}([1: z])=\left[1:(k z)^{-1}\right]$ we find that near $z=0$

$$
\left|d u_{k}\right|^{2}=\frac{u_{k}^{\star} \omega_{2}}{\omega_{1}}=\frac{v_{k}^{\star} \omega_{2}}{\omega_{1}}+\ldots,
$$

with the dots denoting lower order terms. To compute this we find $v_{k}^{\star} d w=-\frac{1}{k z^{2}} d z$ and so

$$
\left|d u_{k}\right|^{2}=\frac{1}{k^{2}|z|^{4}} \frac{\left(1+|z|^{2}\right)}{\left(1+k^{-2}|z|^{-2}\right)^{2}}+\ldots=\frac{1}{k^{2}} \frac{\left(1+|z|^{2}\right)}{\left(k^{-2}+|z|^{2}\right)^{2}}+\ldots
$$

and so

$$
\left|d u_{k}(0)\right|=k \text {, }
$$

which shows that indeed the energy is concentrating at $z=0$. In order to recover the $\pi$ of energy that is there bubbling off, we rescale at $z=0$ with speed $\left|d u_{k}(0)\right|$. Then, we define the blow up sequence

$$
u_{0, k}(z):=u_{k}([1: z / k])=\left[1: 1 / z+u_{\infty}(z / k)\right],
$$

which in compact subsets of $\mathbb{C} \cong T_{0} \Sigma$ uniformly converges to the map $z \mapsto 1 / z$. This can be extended to the degree one map $\mathbb{C P}^{1} \rightarrow M$

$$
u_{0, \infty}([1: z])=[1: 1 / z], \text { with } u_{0, \infty}(0)=\infty,
$$

which accounts for the extra $\pi$ of energy which we had initially lost. Such map is known as the bubble at 0 . We summarize this as a third phenomenon:

(c) Bubbling at the blow up locus, meaning that after appropriately rescaling at the point $0 \in S$, the rescaled maps $u_{0, k}$ converge to a map $u_{0, \infty}: \mathbb{C P}^{1} \rightarrow M$, the bubble, which accounts for the energy loss at the blow up locus. 


\section{Compactness}

This section explains the main aspects behind the standard compactness result for pseudo-holomorphic maps. Our approach is based on ideas from harmonic map theory $[5,7]$.

Theorem 1 (Compactness). Let $(M, \omega, J)$ be a compact symplectic manifold equipped with a compatible almost complex structure and $u_{k}: \Sigma \rightarrow M$ a sequence of pseudo-holomorphic curves with $\left[u_{k}(\Sigma)\right]=\beta \in H_{2}(M, \mathbb{Z})$ fixed. Then, up to passing to a subsequence of $\left\{u_{k}\right\}_{k \in \mathbb{N}}$ which we do not relabel, we have:

(a) There is a finite set $S \subset \Sigma$ so that the maps $u_{k}$ converge uniformly with all derivatives on compact subsets of $\Sigma \backslash S$ to a map $u_{\infty}: \Sigma \backslash S \rightarrow M$.

(b) The map $u_{\infty}$ smoothly extends over $S$ to a pseudo-holomorphic map $u_{\infty}: \Sigma \rightarrow M$, with

$$
\mathcal{E}\left(u_{\infty}\right) \leq \mathcal{E}\left(u_{k}\right)=\langle[\omega], \beta\rangle .
$$

(c) At each point $x \in S$ a pseudo-holomorphic sphere bubbles off, i.e. there is a rescaled subsequence $u_{x, k}$ which uniformly converges with all derivatives on compact subsets of $\mathbb{C} \subset \mathbb{C} \cup\{\infty\}$ to a smooth, nonconstant, pseudo-holomorphic sphere $u_{x}: \mathbb{C P}^{1} \rightarrow M$.

The proof of this theorem will take over the remaining of this section where we shall prove (a) and (b), and the next section where we shall prove (c).

Notation 2. In the rest of this section we shall assume that $(M, \omega, J)$ and $\left\{u_{k}\right\}_{k \in \mathbb{N}}$ are as in the statement of this theorem.

\subsection{Main analytical estimate}

The following local energy estimate is the key ingredient in identifying the blow up set.

Proposition 5 ( $\varepsilon$-regularity). There is $\varepsilon_{0}>0$ and $r_{0}>0$ solely depending on $\Sigma$ with the following significance. Let $u: \Sigma \rightarrow M$ be a pseudo-holomorphic curve, $e=\frac{1}{2}|d u|^{2}$ its energy density and $x \in \Sigma$. Then, for any positive $r \leq r_{0}$

$$
\int_{D_{r}(x)} e \leq \varepsilon<\varepsilon_{0} \Rightarrow \sup _{D_{r / 2}(x)} e \lesssim \frac{1}{r^{2}} \int_{D_{r}(x)} e .
$$

The real proof of this result is given in the Appendix. It relies on a second order inequality for the energy density which we shall show satisfies $\Delta e \lesssim e^{2}$. This should be interpreted in the following way. Whenever a function $e$ satisfies a differential inequality of the kind $\Delta e \lesssim e$ one can write a mean value inequality

$$
\sup _{D_{r / 2}(x)} e \lesssim \frac{1}{r^{2}} \int_{D_{r}(x)} e,
$$

which generalizes the standard mean value property of harmonic functions. However, if the right hand side is to be quadratic in $e$ rather than linear, the same inequality need not hold. For that to be the case, we need $\int e$ to be sufficiently small, which is precisely what the condition $\int_{D_{r}(x)} e \leq \varepsilon<\varepsilon_{0}$ guarantees.

Here we shall give one other different fake argument with the hope of further elucidating why such a result is supposed to elucidate why a result like this should hold.

Fake proof of the $\varepsilon$-regularity. We study the somewhat simpler equation

$$
\Delta e=e^{2},
$$


for simplicity. Using the 2-dimensional Sobolev embedding $L_{2}^{\frac{s}{s+1}} \hookrightarrow L^{s}$, the elliptic inequalities and ignoring the fact that one must shrink the size of the balls we find that

$$
\|e\|_{L^{s}} \lesssim\|e\|_{L_{2}^{\frac{s}{s+1}}} \lesssim\|\Delta e\|_{L^{\frac{s}{s+1}}}+\ldots \lesssim\left\|e^{2}\right\|_{L^{\frac{s}{s+1}}}+\ldots,
$$

where the dots denote higher order terms simply depending on the energy in the ball $B_{r}$. Then, using Hölder's inequality we find that $\left\|e^{2}\right\|_{L^{s+1}} \lesssim\|e\|_{L^{1}}\|e\|_{L^{s}}$ so that

$$
\|e\|_{L^{s}} \lesssim\|e\|_{L^{1}}\|e\|_{L^{s}}+\ldots \lesssim \varepsilon\|e\|_{L^{s}}+\ldots,
$$

where in the last inequality we have used

$$
\|e\|_{L^{1}} \leq \varepsilon<\varepsilon_{0}
$$

be sufficiently small we can reabsorb into the left hand side to get an $L^{s}$-bound on $e$ for all $s>0$. We can not take the limit as $s \nearrow+\infty$ as the involved constant may be blowing up along this limit. However, another use of the elliptic inequalities reveals that for all $s>0$

$$
\|e\|_{L_{2}^{s}} \lesssim\left\|e^{2}\right\|_{L^{s}}+\|e\|_{L^{s}},
$$

with the right hand side being uniformly bounded by the above. Then, taking $s>0$ to be sufficiently large, the Sobolev embedding $L_{2}^{S} \hookrightarrow L^{\infty}$ yields a uniform $L^{\infty}$ bound on $e$.

\subsection{The blow up set is finite}

Let $u_{k}: \Sigma \rightarrow M$ be an infinite sequence of pseudo-holomorphic maps with $\left[u_{k}(\Sigma)\right]=\beta \in H_{2}(M)$ fixed. Then, by the energy formula $\varepsilon\left(u_{k}\right)=\langle[\omega], \beta\rangle$ is uniformly bounded independently of $i$. Let $e_{i}=\frac{1}{2}\left|d u_{i}\right|^{2}$ be the energy density of $u_{i}$ and define the blow up set

$$
S=\bigcap_{r>0}\left\{x \in \Sigma \mid \liminf _{k \rightarrow \infty} \int_{D_{r}(x)} e_{k} \geq \varepsilon_{0}\right\} .
$$

The next result shows that $S$ is a finite set of points. In fact, we shall give an upper bound on its cardinality by bounding its zero dimensional Hausdorff measure.

Lemma 1. In the setting above, the blow up set satisfies $\mathcal{H}^{0}(S) \leq \varepsilon_{0}^{-1}\langle[\omega], \beta\rangle$.

Proof. Then, let $\delta>0$ and consider the cover of $S$ given by $\bigcup_{x \in S} D_{\delta}(x)$. By Vitali's covering lemma we can find a countable subcollection of disks $\left\{D_{\delta}\left(x_{i}\right)\right\}_{i \in I}$ which are pairwise disjoint but such that $\bigcup_{i \in I} D_{5 \delta}\left(x_{i}\right)$ covers $S$. Then, the counting measure $\mathcal{H}^{0}$ of $S$ can be bounded via

$$
\mathcal{H}_{\delta}^{0}(S) \leq \sum_{i \in I}(5 \delta)^{0} \leq \frac{1}{\varepsilon_{0}} \sum_{i \in I} \liminf _{k \rightarrow \infty} \int_{D_{\delta}\left(x_{i}\right)} e_{k} \leq \frac{1}{\varepsilon_{0}} \liminf _{k \rightarrow \infty} \int_{\Sigma} e_{k}=\frac{\langle[\omega], \beta\rangle}{\varepsilon_{0}},
$$

where we have used the fact that the disks $D_{\delta}\left(x_{i}\right)$ are disjoint. As the bound above is independent of $\delta>0$ we find that

$$
\mathcal{H}^{0}(S)=\lim _{\delta \rightarrow 0} \mathcal{H}_{\delta}^{0}(S) \leq \frac{\langle[\omega], \beta\rangle}{\varepsilon_{0}},
$$

as claimed in the statement.

Remark 5. Recall that $\mathcal{E}\left(u_{k}\right)=\langle[\omega], \beta\rangle$ is the energy of each member of the family. As, the estimate above shows that there can at most $\frac{\langle[\omega], \beta\rangle}{\varepsilon_{0}}$ bubbles, we may interpret $\varepsilon_{0}$ as a lower bound on the minimum energy of each bubble. 


\subsection{Convergence away from the blow up set}

Away from the set $S$ defined in 4.1, the $\varepsilon$-regularity result in proposition 5 is the key step in guaranteeing the smooth convergence of the sequence $u_{k} \rightarrow u_{\infty}$ in $\Sigma \backslash S$. We state this as follows.

Lemma 2. There is a pseudo-holomorphic map $u_{\infty}: \Sigma \rightarrow M$ with the property that on compact subsets of $\Sigma \backslash S$ subsequences of the maps $\left\{u_{k}\right\}_{k \in \mathbb{N}}$ uniformly converge with all derivatives to $u_{\infty}$.

Sketch of proof. As $S$ is a finite set of points $\Sigma \backslash S$ is open and any compact $K \subset \Sigma \backslash S$ can be covered with finitely many disks $D_{r}\left(x_{i}\right)$ with $x_{i} \in K$ so that

$$
\liminf _{k \rightarrow \infty} \int_{D_{2 r}(x)} e_{k} \leq \varepsilon<\varepsilon_{0} .
$$

Then, up to passing to a subsequence $\left\{u_{k_{i}}\right\}$, the $\varepsilon$-regularity guarantees that for sufficiently large $k_{i}$

$$
\sup _{y \in K} e_{k_{i}}(y) \lesssim \frac{\varepsilon_{0}}{r^{2}},
$$

yielding a uniform $L^{\infty}$ bound on the family $\left\{e_{k_{i}}\right\}$. As the $\left\{e_{k_{i}}\right\}$ also have a uniform $L^{1}$ bound we conclude that they are uniformly bounded (independently of $k_{i}$ ) in any $L^{p}$ for $p \in[1, \infty]$. Then, we choose bump functions $\chi$ compactly supported in $K$, with $|\nabla \chi| \lesssim 1$, and proceed as follows

$$
\begin{aligned}
\int \chi^{2} e_{k} \Delta e_{k} & =\int d^{\star}\left(\chi^{2} e_{k} d e_{k}\right)+2 \chi e_{k}\left\langle\nabla e_{k} \nabla \chi\right\rangle+\chi^{2}\left|\nabla e_{k}\right|^{2} \\
& =\int 2 \chi e_{k}\left\langle\nabla e_{k} \nabla \chi\right\rangle+\chi^{2}\left|\nabla e_{k}\right|^{2} .
\end{aligned}
$$

Furthermore, completing the square using

$$
\chi^{2}\left|\nabla e_{k}\right|^{2}+2 \chi e_{k}\left\langle\nabla e_{k}, \nabla \chi\right\rangle=\left|\nabla\left(\chi e_{k}\right)\right|^{2}-|\nabla \chi|^{2} e_{k}^{2},
$$

and inserting above we find that ${ }^{3}$

$$
\int\left|\nabla\left(\chi e_{k}\right)\right|^{2}=\int|\nabla \chi|^{2} e_{k}^{2}+\int \chi^{2} e_{k} \Delta e_{k}
$$

Then, using $|\nabla \chi| \lesssim 1$ and $\Delta e_{k} \lesssim e_{k}^{2}$ we find that

$$
\int\left|\nabla\left(\chi e_{k}\right)\right|^{2} \lesssim \int e_{k}^{2}+\int \chi^{2} e_{k}^{3}
$$

By letting $\chi=1$ in a large portion of $K$ we find an $L_{1}^{2}$ bound on $e_{k}$ from an $L^{2}$ and $L^{3}$ bound on $e_{k}$ over all of $K$. Similarly, if we had initially multiplied by $\chi^{2} e_{k}^{p}$ a similar computation would give an $L_{1}^{p+1}$ bound on $e_{k}$ from its $L^{p+1}$ and $L^{p+2}$ bounds. It is also possible to obtain analogous estimates on all higher derivatives of $e_{k}$ by differentiating the pseudo-holomorphic curve equation and finding inequalities for $\Delta\left|\nabla^{l} d u_{k}\right|^{2}$ instead of $\Delta e_{k}$. All this gives sufficiently strong bounds on the maps $u_{k}$ to guarantee that all their derivatives in $K$ are equicontinuous. At this point, a similar application of Ascoli-Arzelá type theorems, in the form of compact Sobolev embeddings, as that we used for geodesics in section 3.1 yields the stated convergence of the maps $u_{k}$.

Remark 6. The proof of parts (a) in Theorem 1 is now complete. Part (b) follows from the removable singularities criteria stated in Proposition 4. The only subtlety in applying it is that we must guarantee that the map $u_{\infty}$ is pseudo-holomorphic. That follows from the fact that the $u_{k}$ are converging to it with all derivatives.

3 This formula, which follows simply from the Leibniz rule, is sometimes called the Agmon trick and I learned it from Mark Stern, see [1] and [2]. 


\section{Bubbling}

The following result proves part (c) of Theorem 1 by showing that at each point of the blow up set $S$ there is a pseudo-holomorphic sphere bubbling off. This is usually called the "inner bubble" at $x$. In general there may be a bubble tree ${ }^{4}$ forming and we need to proceed in a more careful manner to see the different bubbles.

Lemma 3. Let $x \in S$, then there is a sequence of points $x_{k} \rightarrow x$ and a nullsequence $\delta_{k}$ such that $u_{x_{k}}(z):=$ $u\left(\exp _{x_{k}}\left(\delta_{k} z\right)\right)$ converges uniformly on compact subsets of $\mathbb{C} \subset \mathbb{C} \cup\{\infty\}$ to a non-constant pseudo-holomorphic sphere

$$
u_{x}: \mathbb{C P}^{1} \rightarrow M \text {. }
$$

Proof. Let $x \in S$, then for all $r>0$

$$
\liminf _{k \rightarrow \infty} \int_{D_{r}(x)} e_{k} \geq \varepsilon_{0},
$$

which implies the existence of a sequence of points $\left\{x_{k}\right\}_{k \in \mathbb{N}}$ with $x_{k} \rightarrow x$ and $e_{k}\left(x_{k}\right) \nearrow+\infty$. Then, we fix the nullsequence $\delta_{k}=\left|d u_{k}\left(x_{k}\right)\right|^{-1}=\left(2 e_{k}\left(x_{k}\right)\right)^{-1 / 2} \rightarrow 0$ and define

$$
u_{x_{k}}:=s_{x_{k}}^{\star} u_{k}, g_{x_{k}}=\delta_{k}^{-2} s_{x_{k}}^{\star} g,
$$

where $s_{x_{k}}(z)=\exp _{x_{k}}\left(\delta_{k} z\right) .{ }^{5}$ We now work in an open set of $\mathbb{C}$ which we identify with all the tangent spaces $T_{x_{k}} \Sigma$ and consider the energy density

$$
e_{x_{k}}=\frac{1}{2}\left|d u_{x_{k}}\right|_{x_{x_{k}}}^{2}
$$

of this rescaled subsequence. By construction, i.e. the choice of the $\delta_{k}$ we have that

$$
e_{x_{k}}(0)=\frac{\delta_{k}^{2}}{2}\left|d u_{k}\left(x_{k}\right)\right|_{g}^{2}=\frac{1}{2}>0 .
$$

We conclude that the sequence $u_{x_{k}}: D_{r / \delta_{k}}(0) \rightarrow M$, if converging, cannot converge to a constant map. We must now prove that indeed this sequence converges. For this we must put a further restriction on the points $x_{k}$ which selects the "inner bubble". We choose $x_{k}$ not only imposing that $\delta_{k}=\left|d u_{k}\left(x_{k}\right)\right|^{-1} \nearrow+\infty$ but we actually choose an $x_{k}$ at which the maximum of $\left|d u_{k}\right|$ is achieved in $\bar{D}_{r}(x)$, i.e.

$$
\left|d u_{k}\left(x_{k}\right)\right|=\sup _{y \in \bar{D}_{r}(x)}\left|d u_{k}(y)\right| .
$$

Then, we immediately find that

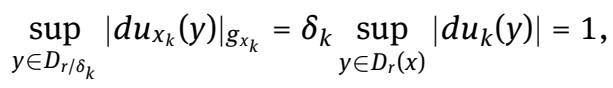

which is uniformly bounded. Then, by the proof of Lemma 2, a subsequence of $\left\{u_{x_{k}}\right\}_{k \in \mathbb{N}}$ converges uniformly with all derivatives on compact sets of $\mathbb{C}$ to a nonconstant map

$$
u_{x}: \mathbb{C} \rightarrow M .
$$

Its energy can be bounded by the fact that the energy of each element in the sequence satisfies

$$
\int_{D_{r / \delta_{k}}(0)} e_{x_{k}} \operatorname{vol}_{g_{x_{k}}}=\int_{D_{r / \delta_{k}}(0)} s_{x_{k}}^{\star}\left(e_{k} \operatorname{vol}_{\Sigma}\right)=\int_{D_{r}(x)} e_{k} \operatorname{vol}_{\Sigma} \leq\langle[\omega], \beta\rangle .
$$

\footnotetext{
4 Meaning bubbles within the bubbles within bubbles and so on.

5 We regard these as being defined in disks of radius $\delta_{k}^{-1} r$ inside $\mathbb{C} \cong T_{x_{k}} \Sigma$. Notice in particular that the metrics $g_{x_{k}}$ geometrically converges to the Euclidean one in compact subsets of $\mathbb{C} \cong T_{x_{k}} \Sigma$.
} 
This gives a $k$ independent upper bound for the energy of each $u_{x_{k}}$. Thus, the map $\tilde{u}_{x}: \mathbb{C P}^{1} \rightarrow M$ given by $\tilde{u}_{x}(z)=u_{x}(1 / z)$ has finite energy and by removal of singularities stated in proposition 4 we find that this extends to a smooth pseudo-holomorphic map $\tilde{u}_{x}: \mathbb{C P}^{1} \rightarrow M$. Given that the map $z \mapsto 1 / z$ is a biholomorphism we find that also $u_{x}$ extends to a pseudo-holomorphic sphere.

Remark 7 (Noncompact domain curves). When $\Sigma$ is a strip, as for example in Lagrangian Floer homology, bubbling at boundary points happens in codimension 1. When $\Sigma$ is a cylinder, as in Hamiltonian Floer homology, bubbling can happen along the ends.

\section{Transversality}

As in the introduction, in this section we view the moduli space of pseudo-holomorphic curves as the zero locus of a section $\bar{\partial}_{J}$ of the bundle $\mathcal{T} \rightarrow C^{\infty}(\Sigma, M)$, whose fiber at $u$ is $\mathcal{T}_{u}=C^{\infty}\left(\Sigma, \Lambda^{0,1} \Sigma \otimes u^{\star} T M\right)$. Now, let $u$ be a pseudo-holomorphic map and differentiate $\bar{\partial}_{J}$ at $u$, this gives a map $d_{u} \bar{\partial}_{J}: T_{u} C^{\infty}(\Sigma, M) \rightarrow T_{(u, 0)} \mathcal{T}$, which operates on $\chi \in C^{\infty}\left(\Sigma, u^{\star} T M\right)$ via

$$
\begin{aligned}
d_{u} \bar{\partial}_{J}(\chi) & =\left.\frac{d}{d t}\right|_{t=0} \bar{\partial}_{J}(u+t \chi) \\
& =\frac{1}{2}\left(d \chi+J_{u} d \chi \circ j+\left(d J_{u}\right)(\chi) d u \circ j\right) .
\end{aligned}
$$

Decomposing $T_{(u, 0)} \mathcal{T}$ as $C^{\infty}\left(\Sigma, u^{\star} T M\right) \oplus C^{\infty}\left(\Sigma, \Lambda^{0,1} \Sigma \otimes u^{\star} T M\right)$ and projecting $d_{u} \bar{\partial}_{J}$ on the second we obtain a map

$$
D_{u} \bar{\partial}_{J}: C^{\infty}\left(\Sigma, u^{\star} T M\right) \rightarrow C^{\infty}\left(\Sigma, \Lambda^{0,1} \Sigma \otimes u^{\star} T M\right),
$$

given by

$$
\begin{aligned}
D_{u} \bar{\partial}_{J}(\chi) & =\frac{1}{2}\left(d_{u} \bar{\partial}_{J}+J_{u} d_{u} \bar{\partial}_{J} \circ j\right) \\
& =\frac{1}{4}\left(d \chi+J_{u} d \chi \circ j+\left(d J_{u}\right)(\chi) d u \circ j\right)+\frac{1}{4} J_{u}\left(d \chi+J_{u} d \chi \circ j+\left(d J_{u}\right)(\chi) d u \circ j\right) \circ j \\
& =\frac{1}{2}\left(d \chi+J_{u} d \chi \circ j\right)+\frac{1}{4}\left(\left(d J_{u}\right)(\chi) d u \circ j-J_{u}\left(d J_{u}\right)(\chi) d u\right) \\
& =\bar{\partial}_{J_{u}} \chi-\frac{1}{2} J_{u}\left(d J_{u}\right)(\chi) d u
\end{aligned}
$$

where in the two last equalities we respectively used the Cauchy-Riemman equation $J_{u} d u=d u \circ j$, and $J^{2}=-1$ to compute that $J_{u} d J_{u}+d J_{u} J_{u}=0$.

In order to think of $D_{u} \bar{\partial}_{J}$ of a smooth map between Banach manifolds we must topologize the relevant spaces with Banach space norms. Ideally we would like to work with smooth maps, however the $C^{\infty}$-topology is not associated with a norm ${ }^{6}$ and instead we fix $k \in \mathbb{N}$ and $p>0$ so that $k p>2$ and regard $D_{u} \bar{\partial}_{J}$ as a map

$$
D_{u} \bar{\partial}_{J}: L_{k}^{p}\left(\Sigma, u^{\star} T M\right) \rightarrow L_{k-1}^{p}\left(\Sigma, \Lambda^{0,1} \Sigma \otimes u^{\star} T M\right) .
$$

A word must be said about the reason for having $k p>2$. The main reasons for doing this are: First, in this range the Rellich-Kondrachov compactness theorem yields a compact embedding $L_{k}^{p} \hookrightarrow C^{0}$. Secondly, in this range the $L_{k}^{p}$ spaces form an algebra which is useful in dealing with the non-linearities. We shall now sketch the proof the following result.

Proposition 6 (The linearised operator is Fredholm). Let $u$ be a pseudo-holomorphic map and for a compatible almost complex structure J of class $C^{l}$ with $l \geq k$. Then, the operator 6.1 is Fredholm of index

$$
\text { index }\left(D_{u} \bar{\partial}_{J}\right)=n \chi(\Sigma)+2\left\langle c_{1}(T M), u[\Sigma]\right\rangle .
$$

6 It is conceivable that one could work in the $C^{\infty}$ setting by using Fréchet spaces. 
Proof. First we prove that the operator $J_{u}\left(d J_{u}\right)(\cdot): L_{k}^{p} \rightarrow L_{k-1}^{p}$ is compact under the stated hypothesis (this is where the condition that $l \geq k$ shows up). As the Cauchy-Riemann operator $\bar{\partial}_{J_{u}}$ is elliptic, it is Fredholm between the stated function spaces. Having in mind that $D_{u} \bar{\partial}_{J}=\bar{\partial}_{J_{u}}-\frac{1}{2} J_{u}\left(d J_{u}\right)$ and that the Fredholm index is invariant by compact perturbation we conclude that $D_{u} \bar{\partial}_{J}$ is Fredholm of index index $\left(D_{u} \bar{\partial}_{J}\right)=\operatorname{index}\left(\bar{\partial}_{J_{u}}\right)$ which one can compute using the Hirzebruch-Riemann-Roch formula.

Let $\beta \in H_{2}(M, \mathbb{Z})$ be an homology class in $M$ and define the moduli space of somewhere injective parametrized pseudo-holomorphic curves in the class $\beta$, denoted $\mathcal{M}^{\star}(\beta, J)$ by

$$
\left\{u:(\Sigma, j) \rightarrow(M, J) \mid \bar{\partial}_{J} u=0, u([\Sigma])=\beta, \exists_{s \in \Sigma} d u_{s} \neq 0 \text { and } u^{-1}(u(s))=s\right\} .
$$

The condition that $u$ be somewhere injective implies that the map $u$ does not factor through some another curve multiply (branched) covered by $\Sigma$.

Theorem 2 (Transversality). For generic smooth almost complex structures $J$, the moduli space $\mathcal{M}^{*}(\alpha, J)$ is a finite dimensional $2 c_{1}(M)[\beta]+2 n(1-g)$ real manifold.

\section{Main example of application: The nonsqueezing theorem}

In this section we will give a self contained proof of Gromov's nonsequeezing theorem by simply making use of the results we have so far presented. For the original proof whose argument we follow see the [4]. There are also several other very good expositions of the nonsqeezing theorem containing all the details, see for example [6] and [8]. Before stating the result we introduce the main characters, the symplectic $r$-ball and the symplectic $R$-cylinder. These are are

$$
B_{r}^{2 n}:=\left\{\left(x_{i}, y_{i}\right) \in \mathbb{R}^{2 n} \mid \sum_{i=1}^{n}\left(\left|x_{i}\right|^{2}+\left|y_{i}\right|^{2}\right)<r^{2}\right\},
$$

which is the radius $r$ ball equipped with the induced canonical symplectic structure $\omega_{0}=\sum_{i=1}^{n} d x_{i} \wedge d y_{i}$ and

$$
C_{R}^{2 n}:=\left\{\left.\left(x_{i}, y_{i}\right) \in \mathbb{R}^{2 n}|| x_{1}\right|^{2}+\left|y_{1}\right|^{2}<r^{2}\right\}
$$

which is the radius $R$ cyclinder, again equipped with the induced canonical symplectic structure. Now, we state the theorem.

Theorem 3 (Gromov's nonsqueezing). There is a symplectic embedding

$$
i: B_{r}^{2 n} \hookrightarrow C_{R}^{2 n},
$$

if and only if $r \leq R$.

One direction in proving this theorem is quite straightforward as, for $r \leq R$, the standard inclusion $B_{r}^{2 n} \hookrightarrow C_{R}^{2 n}$ is symplectic. Hence, we need only prove the direction asserting that if a symplectic embedding $i: B_{r}^{2 n} \hookrightarrow C_{R}^{2 n}$ exists then $r \leq R$. Starting from the existence of such a symplectic embedding $i$, the image $i\left(B_{2}^{2 n}\right)$ is bounded in $C_{R}^{2 n}$ and so, for any $\delta>0$ we may compactify it inside

$$
M=\mathbb{S}_{R+\delta}^{2} \times \mathbb{T}^{2 n-2},
$$

where $\mathbb{T}^{2 n-2}$ is a sufficiently large torus and $\mathbb{S}_{R+\delta}^{2}$ a the 2-sphere of radius $R+\delta$. Then, we equip $M$ with an almost complex structure $J$ which in the image of $i$ agrees with the push-forward of the standard one on $B_{r}^{2 n}$.

Lemma 4. There a pseudo-holomorphic curve $u: \mathbb{C P}^{1} \rightarrow M$ with $u\left(\mathbb{C P}^{1}\right) \in\left[S^{2} \times 0\right]$ passing through $i(0)$. 
Proof. Let $\beta:=\left[S^{2} \times 0\right]$. Then, for any other almost complex structure $J^{\prime}$, the moduli spaces $\mathcal{M}^{\star}(\beta, J)$ and $\mathcal{M}^{*}\left(\beta, J^{\prime}\right)$ are cobordant. Furthermore, as the class $\beta$ generates $\pi_{2}(M)$, the pseudo-holomorphic curves in the class $\beta$ have minimal energy and so bubbling cannot occur. By this reason, the evaluation maps

$$
\mathrm{ev}_{J^{\prime}}: \mathcal{M}^{\star}\left(\beta, J^{\prime}\right) \times_{\mathrm{PSL}(2, C)} \mathbb{C P}^{1} \rightarrow \mathbb{C P}^{1},[u, z] \mapsto u(z),
$$

and the similarly defined $\operatorname{ev}_{J} \mathcal{M}^{*}(\beta, J) \times_{\mathrm{PSL}(2, C)} \mathbb{C P}^{1}$, have the same degree. When $J^{\prime}$ is the standard almost complex structure on $M$, the degree of $\mathrm{ev}_{J^{\prime}}$ can easily be computed as follows. Any $u \in \mathcal{M}^{*}\left(\beta, J^{\prime}\right)$ can be written as

$$
u(z)=(\psi(z), t),
$$

for $\psi \in \operatorname{PSL}(2, C)$ and $t \in \mathbb{T}^{2 n-2}$. This accounts for all the $2 n+4$-dimensions in the moduli space and so it describes it fully. Furthermore, in this case $\mathrm{ev}_{J^{\prime}}$ has degree 1 and as this is invariant by cobordisms $\operatorname{deg}\left(\mathrm{ev}_{J}\right)=$ 1. Hence, there is $u \in \mathcal{M}^{\star}(\beta, J)$ with $\operatorname{ev}_{J}[u, 0]=i(0)$, i.e. a pseudo-holomorhic curve as in the statement.

Such a pseudo-holomorphic curve has image $C \subset M$ with $\operatorname{Area}(C)=\mathcal{E}(u)=\pi(R+\delta)^{2}$. On the other hand, pulling $C \cap i\left(B_{r}^{2 n}\right)$ back to the ball we find that

$$
\operatorname{Area}\left(i^{-1}(C) \cap B_{r}(0)\right) \geq \pi r^{2},
$$

as the minimal area minimal surface passing through the origin is the disk of area $\pi r^{2}$. Hence, $\pi r^{2} \leq \pi(R+\delta)^{2}$ for all $\delta>0$ finishing the proof of the nonsqueezing theorem.

\subsection{Acknowledgments}

The author is grateful for the comments and suggestions of an anonymous referee which helped him improve the exposition.

The author was supported by Fundação Serrapilheira (grant 1812-27395), by CNPq grants 428959/2018-0 and 307475/2018-2, and FAPERJ through the program Jovem Cientista do Nosso Estado E-26/202.793/2019.

\section{A Proof of the Weitzenböck formula and $\varepsilon$-regularity}

This section follows the analogous results in [6] apart from minor rearrangements in the arguments and their exposition.

\section{A.1 Weitzenböck formula}

Let $u: \Sigma \rightarrow M$ be a pseudo-holomorphic curve and $e: \Sigma \rightarrow \mathbb{R}$ the associated energy density defined by $e=\frac{1}{2}|d u|^{2}$, see remark 2 . We shall now prove the following differential inequality which is key in showing the basic estimates.

Lemma 5 (Weitzenböck formula). The energy density satisfies

$$
\Delta e \lesssim e^{2}
$$

Proof. Keeping in mind that $\partial_{x} u+J \partial_{y} u=0$ so that $\left|\partial_{x} u\right|=\left|\partial_{y} u\right|$ and $e=\left|\partial_{x} u\right|^{2}$ we compute

$$
\Delta e=\left\langle\partial_{x} u, \nabla^{\star} \nabla \partial_{x} u\right\rangle-\left|\nabla \partial_{x} u\right|^{2},
$$


Using the notation $\nabla_{x}=\nabla_{\partial_{x} u}$, and similarly for $y$, we find

$$
\begin{aligned}
\nabla^{\star} \nabla \partial_{x} u & =\nabla_{x} \nabla_{x} \partial_{x} u+\nabla_{y} \nabla_{y} \partial_{x} u \\
& =\nabla_{x} \nabla_{x}\left(-J \partial_{y} u\right)+\nabla_{y} \nabla_{x} \partial_{y} u \\
& =\nabla_{x}\left(\nabla_{x}\left(-J \partial_{y} u\right)+\nabla_{y} \partial_{y} u\right)+\left(\nabla_{y} \nabla_{x}-\nabla_{x} \nabla_{y}\right) \partial_{y} u \\
& =\nabla_{x}\left(\nabla_{x}\left(-J \partial_{y} u\right)+\nabla_{y}\left(J \partial_{x} u\right)\right)+R\left(\partial_{y} u, \partial_{x} u\right) \partial_{y} u \\
& =\nabla_{x}\left(\left(\nabla_{y} J\right) \partial_{x} u-\left(\nabla_{x} J\right) \partial_{y} u\right)+R\left(\partial_{y} u, \partial_{x} u\right) \partial_{y} u .
\end{aligned}
$$

Thus, we find that

$$
\Delta e \leq\left|\partial_{x} u\right|\left(I_{x, y, x}+I_{x, x, y}\right)+\operatorname{Riem}\left(\partial_{y} u, \partial_{x} u, \partial_{y} u, \partial_{x} u\right)-\left|\nabla \partial_{x} u\right|^{2},
$$

with $I_{x, y, x}:=\left|\nabla_{x}\left(\left(\nabla_{y} J\right) \partial_{x} u\right)\right|$ and $I_{x, x, y}$ defined similarly. Now we separately bound the terms $I_{x, y, x}$ and $I_{x, x, y}$. For the first of these we find

$$
\begin{aligned}
I_{x, y, x} & \leq\left|\nabla_{x}\left(\nabla_{y} J\right) \partial_{x} u\right|+\left|\left(\nabla_{y} J\right) \nabla_{x} \partial_{x} u\right| \\
& \leq\left(\left|\nabla_{x} \partial_{y} u\right|+\left|\partial_{x} u\right|\left|\partial_{y} u\right|\right)\left|\partial_{x} u\right|+\left|\partial_{y} u\right|\left|\nabla_{x} \partial_{x} u\right| \\
& \leq\left(\left|\nabla_{x} \partial_{y} u\right|+\left|\nabla_{x} \partial_{x} u\right|\right) e^{1 / 2}+e^{3 / 2},
\end{aligned}
$$

while for the second we have

$$
\begin{aligned}
I_{x, x, y} & \leq\left|\nabla_{x}\left(\nabla_{x} J\right) \partial_{y} u\right|+\left|\left(\nabla_{x} J\right) \nabla_{x} \partial_{y} u\right| \\
& \leq\left(\left|\nabla_{x} \partial_{x} u\right|+\left|\partial_{x} u\right|\left|\partial_{x} u\right|\right)\left|\partial_{y} u\right|+\left|\partial_{x} u\right|\left|\nabla_{x} \partial_{y} u\right| \\
& \leq\left(\left|\nabla_{x} \partial_{x} u\right|+\left|\nabla_{x} \partial_{y} u\right|\right) e^{1 / 2}+e^{3 / 2} .
\end{aligned}
$$

From these we find that

$$
\begin{aligned}
\left|\partial_{x} u\right|\left(I_{x, y, x}+I_{x, x, y}\right) & \leq \frac{1}{2}\left(\left|\nabla_{x} \partial_{y} u\right|^{2}+\left|\nabla_{x} \partial_{x} u\right|^{2}+\left|\nabla_{x} \partial_{x} u\right|^{2}+\left|\nabla_{x} \partial_{y} u\right|^{2}\right)+4 e^{2} \\
& \leq\left|\nabla_{x} \partial_{x} u\right|^{2}+\left|\nabla_{y} \partial_{x} u\right|^{2}+4 e^{2},
\end{aligned}
$$

and given that $\operatorname{Riem}\left(\partial_{y} u, \partial_{x} u, \partial_{y} u, \partial_{x} u\right) \leq K e^{2}$, where $K$ denotes the supremum over all sectional curvatures. Thus, $\Delta e \lesssim(4+K) e^{2}$ and the result follows.

\section{A.2 $\varepsilon$-regularity}

Lemma 6. Let $c>0$, then there is $\varepsilon_{0}>0$ such that any smooth function $e: D_{r} \subset \mathbb{R}^{2} \rightarrow \mathbb{R}$ so that $\Delta e \leq c e^{2}$

$$
\int_{D_{r}} e \leq \varepsilon<\varepsilon_{0} \Rightarrow \sup _{D_{r / 2}} e \lesssim \frac{1}{r^{2}} \int_{D_{r}} e .
$$

Proof. We use the Heinz trick and consider the function $\theta:[0, r] \rightarrow \mathbb{R}$ defined by $\theta(s)=(r-s)^{2} \sup _{D_{s}} e$. This function is continuous, nonnegative, and vanishes at $s=r$ so it has an interior maximum $s \star \in(0, r)$ and we denote by $z_{\star}$ the point in $D_{S_{\star}}$ at which $\sup _{D_{s_{\star}}} e$ is achieved. Then,

$$
\theta\left(s_{\star}\right)=\left(r-s_{\star}\right)^{2} e\left(z_{\star}\right),
$$

and for $\delta=\frac{r-s_{\star}}{2}$ we have that $D_{\delta}\left(z_{\star}\right) \subset D_{\delta+S_{\star}} \subset D_{r}$. For points $z \in D_{\delta}\left(z_{\star}\right)$ we have

$$
\sup _{D_{\delta}\left(z_{\star}\right)} e \leq \sup _{D_{\delta+s_{\star}}} e=\left(r-s_{\star}-\delta\right)^{-2} \theta\left(\delta+s_{\star}\right) \leq 4\left(r-s_{\star}\right)^{-2} \theta\left(s_{\star}\right)=4 e\left(z_{\star}\right) .
$$

Then, inside $D_{\delta}\left(z_{\star}\right)$ we have

$$
\Delta e \leq c e^{2} \leq 16 c e(z \star)^{2} .
$$


As $\Delta \operatorname{dist}\left(\cdot, z_{\star}\right)^{2}=-4$, we find that

$$
\Delta\left(e+4 c e\left(z_{\star}\right)^{2} \operatorname{dist}\left(\cdot, z_{\star}\right)^{2}\right) \leq 0,
$$

and the mean value inequality yields

$$
e\left(z_{\star}\right) \leq \frac{1}{\pi s^{2}} \int_{D_{s}\left(z_{\star}\right)} e+4 c e\left(z_{\star}\right)^{2} \operatorname{dist}\left(\cdot, z_{\star}\right)^{2},
$$

for all $s \leq \delta$. Carrying out the integration above yields

$$
e\left(z_{\star}\right) \leq 2 c e\left(z_{*}\right)^{2} s^{2}+\frac{1}{\pi s^{2}} \int_{D_{s}\left(z_{*}\right)} e .
$$

Now, we show that there is $\varepsilon_{0}>0$, which we shall then fix, so that $e\left(z_{\star}\right) \leq \varepsilon_{0} / \delta^{2}$. Suppose not, then $\delta^{2}>$ $\varepsilon_{0} / e\left(z_{\star}\right)$ and we can choose $s^{2}=\varepsilon_{0} / e\left(z_{\star}\right)$ in the inequality above. This gives that

$$
e\left(z_{\star}\right) \leq 2 c \varepsilon_{0} e\left(z_{\star}\right)+\frac{e\left(z_{\star}\right)}{\pi \varepsilon_{0}} \int_{D_{\delta}\left(z_{\star}\right)} e,
$$

which for $\frac{\pi-1}{\pi} \frac{1}{2 c}<\varepsilon_{0}<\frac{1}{2 c}$ gives

$$
\int_{D_{r}} e \geq \int_{D_{\delta}(z *)} e \geq \varepsilon_{0}
$$

contradicting the hypothesis in the statement. Hence, we must have $e\left(z_{\star}\right) \leq \varepsilon_{0} / \delta^{2}$, i.e. $\delta^{2} \leq \varepsilon_{0} / e\left(z_{\star}\right)$ from which we find that

$$
e\left(z_{\star}\right) \leq 2 c \varepsilon_{0} e\left(z_{\star}\right)+\frac{1}{\pi s^{2}} \int_{D_{s}\left(z_{*}\right)} e .
$$

Then, finally setting $\varepsilon_{0}=\frac{5}{12 c}$ we find that

$$
e\left(z_{\star}\right) \leq \frac{6}{\pi s^{2}} \int_{D_{s}\left(z_{\star}\right)} e .
$$

As this is valid for all $s \leq \delta$ we may as well rewrite it as

$$
e\left(z_{\star}\right) \leq \frac{6}{\pi \delta^{2}} \int_{D_{\delta}\left(z_{*}\right)} e .
$$

Returning to the definition of $\theta, s_{\star}, z_{\star}$, we find that

$$
e(0)=\frac{\theta(0)}{r^{2}} \leq \frac{\theta\left(s_{\star}\right)}{r^{2}}=\frac{4 \delta^{2} e\left(z_{\star}\right)}{r^{2}} \leq \frac{24}{\pi r^{2}} \int_{D_{\delta}\left(z_{\star}\right)} e \leq \frac{24}{\pi r^{2}} \int_{D_{r}} e,
$$

as we wanted to show.

\section{References}

[1] Agmon, Shmuel. Lectures on exponential decay of solutions of second-order elliptic equations: Bounds on eigenfunctions of $n$-body Schrodinger operations.(mn-29). Vol. 29. Princeton University Press, 2014.

[2] Degeratu, A., Stern, M. Witten Spinors on Nonspin Manifolds. Commun. Math. Phys. 324, 301-350 (2013).

[3] S. Donaldson, Karen Uhlenbeck and the Calculus of Variations, March 2019, Notices of the American Mathematical Society 66(03):1 DOI: 10.1090/noti1806

[4] M. Gromov, Pseudo holomorphic curves in symplectic manifolds. Inventiones mathematicae 82.2 (1985): 307-347.

[5] Lin, Fang-Hua, and Tristan Riviere. Energy quantization for harmonic maps. Duke Mathematical Journal 111.1 (2002): 177-193.

[6] D. McDuff, and D. Salamon, J-holomorphic curves and symplectic topology. Vol. 52. American Mathematical Soc., 2012.

[7] Sacks, J., and K. Uhlenbeck. The existence of minimal immersions of two-spheres. Bulletin of the American Mathematical Society 83.5 (1977): 1033-1036.

[8] Wendl, Chris. Lectures on holomorphic curves in symplectic and contact geometry. arXiv preprint arXiv:1011.1690 (2010). 